\title{
Factors Affectıng Sentinel Lymph Node Metastasis in Patients with Breast Cancer Undergoing Sentinel Lymph Node Biopsy before or after Systemic Therapy
}

\author{
Mehmet Furkan Sağdiç ${ }^{1}$, Cemil Yüksel ${ }^{1}$, Serdar Çulcu ${ }^{1, *}$, Bülent Aksel ${ }^{1}$, Cihangir Özaslan ${ }^{1}$, Suat Kutun ${ }^{1}$ and Lütfi \\ Doğan $^{1}$
}

1University of Health Sciences, Ankara Abdurrahman Yurtaslan Oncology Training and Research Hospital, Ankara, Turkey

* Corresponding author: Serdar Culcu, University of Health Sciences, Ankara Abdurrahman Yurtaslan Oncology Training and Research Hospital, Ankara, Turkey. Tel: +90 5054634723; Email: serdarculcu@gmail.com

Received 2021 April 29; Revised 2021 0ctober 22; Accepted 2021 0ctober 29

\begin{abstract}
Background: The tendency to spread to sentinel lymph node (SLN) may differ depending on the biological, clinical, and histopathological features of tumors. If the factors that affect SLN metastasis (SLNM) are known, there may be no need to perform SLN biopsy (SLNB) in some groups.

Objectives: This study aimed to investigate the factors affecting SLNM in patients who underwent surgery and SLNB before (surgery group) or after (neoadjuvant chemotherapy group) systemic therapy in the light of current biological characteristics of tumors and patients.

Methods: The study included patients who were operated on for breast cancer and underwent SLNB in our institute between 2017 and 2019. The study included a total of 1,050 patients, who were divided into the surgery ( $n=900)$ and neoadjuvant chemotherapy (NAC) groups $(n=150)$. The patients' tumor localization, tumor size, histological subtype, grade, receptor status, lymphovascular invasion (LVI) status, the number of sentinel lymph nodes removed, metastatic lymph nodes in SLNB, and axillary dissection status were analyzed in this study.

Results: The study included a total of 1,050 patients, who were assigned to the surgery ( $\mathrm{n}=900)$ and NAC groups (n=150). Of the patients, $311(34.5 \%)$ cases had SLNM. In the surgery group, multivariate analyses showed that grade III, LVI, Her2 (+) increased the risk of metastasis. In the NAC group, the analyses showed Pre-NAC clinical findings of LN metastasis and luminal A subtypes as effective factors. The factors affecting SLNM were analyzed, and the univariate analyses showed that grades II and III, a tumor size of $>2 \mathrm{~cm}$, LVI, Her2 ( + ), and triple negative increased the risk of metastasis. The analyses also revealed LVI as the most important risk factor for SLN metastasis. Conclusion: Knowing the factors affecting SLNM can provide clues for the type of intervention, reconstruction, and radiotherapy planning of patients to be operated on directly or after NAC. In our study, it was found that patient age, tumor size, tumor biology, tumor grade, and especially LVI status were very important in predicting SLN positivity. It is believed that these features should be taken into account when determining the treatment strategy.
\end{abstract}

Keywords: Breast cancer, Neoadjuvant therapy, Sentinel lymph node

\section{Background}

The status of axillary lymph node (ALN) along with clinical and histopathological factors is considered an important prognostic factor in invasive breast cancer. Sentinel lymph node biopsy (SLNB) is a routine method used in the surgical treatment of eligible breast cancer patients for regional staging $(1,2)$ The SLN, which is the first station of tumor infiltration through lymphatic drainage, provides information about the condition and prognosis of the axillary region and prevents unnecessary axillary lymph node dissection (ALND) in patients without SLN metastasis (SLNM). In addition, it has been shown that performing ALND is not required in patients with $\mathrm{T}_{1}-\mathrm{T}_{2}$ tumors who have 2 or less SLNM and who will undergo breast-conserving surgery and receive radiotherapy (RT) (3). The views suggesting that the spread of breast cancer to SLN and non-SLN are two biologically different concepts. The status of SLN has become an extremely important determining factor of treatment today. The tendency to spread to SLN may differ depending on the biological, clinical, and histopathological features of tumors. The necessity of performing SLNB in patients with a low SLNM risk has been questioned $(4,5)$. If the factors that affect SLNM are known, there may be no need to perform SLNB in some groups. The response to axillary metastasis with systemic treatment also varies in tumors of different characteristics. Axillary pathological complete response is higher after neoadjuvant therapy in triple negative (TN) and Her2 (+) tumors (6). In the near future, it can be predicted that there will be some histopathological, molecular, and biological characteristic features in locoregional therapy instead of conventional prognostic factors.

\section{Objectives}

This study aimed to investigate the factors affecting SLNM in patients who underwent surgery and SLNB before (surgery group) or after (neoadjuvant chemotherapy (7) group) systemic therapy in the light of current biological characteristics of tumors and patients. In this way, it is aimed to shed light on studies to be conducted to identify a group of patients who will not require SLNB. 


\section{Methods}

This retrospective study included patients who were operated on for breast cancer and underwent SLNB in the general surgery clinic of our institute between January 2017 and August 2019. The study included a total of 1,050 patients, who were divided into the surgery $(n=900)$ and NAC $(n=150)$ groups. Patients with confirmed breast cancer by core needle (tru-cut) biopsy were analyzed. On the other hand, the patients with de-novo distant metastasis, pure ductal carcinoma in-situ, malignancy other than breast cancer, previous breast or axillary surgery, male and pregnant patients, patients who underwent SLNB without previous fine-needle aspiration biopsy (FNAB) despite suspected clinical axillary metastasis, and those with incomplete data and without regular follow-up were excluded from the study.

The missing data in the tru-cut biopsy were obtained from the final pathology reports. In addition to the patients without clinical (physical examination, axillary ultrasound guided) findings of axillary lymph node metastasis (ALNM), those with clinically suspected axillary metastases but whose suspected axillary metastasis was excluded by FNAB and who underwent SLNB were directly evaluated in the surgery group. The patients with and without pre-NAC clinical findings of axillary metastasis were included in the NAC group. The patients with a post-NAC stage of cN0 underwent SLNB.

The patients' age, menstrual status, breast and tumor localization, tumor size, histological subtype, grade, receptor status (estrogen receptor, progesterone receptor, and Cerb-B2, Ki-67), luminal subtype, lymphovascular invasion (LVI) status, the number of sentinel lymph nodes removed, SLN status, number of metastatic lymph nodes in SLNB, and axillary dissection status were analyzed in this study.

The presence of tumor cells in the blood and lymphatic vessels, the presence of tumor cells under the endothelium lining the vascular channels, and the detection of the invasion of the vascular wall or endothelium by tumor cells were considered LVI. Hormone receptor positivity of $1 \%$ or above was considered hormone receptor (HR) (+). In SLN, metastases between 0.2 and $2 \mathrm{~mm}$ were considered micro-metastasis, while metastases smaller than 0.2 mm or less than 200 tumor cells were considered isolated tumor cells (ITC). The presence of micrometastasis and ITC in the patients who directly underwent surgical intervention was considered SLN (-), while it was considered SLN (+) in patients who received NAC. Gene amplification was performed using fluorescein in situ hybridization in patients with Her2/neu+2, and neoadjuvant antiHer2 therapy was administered to all Her2 (+) patients in the NAC group. None of the patients underwent SLNB before NAC.

All patients underwent SLNB with the combined technique. The radioactive material injection was performed a day before or on the morning of the surgery. Periareolar methylene blue injection was performed intraoperatively. Lymph nodes with radioactivity uptake on gamma counter, stained blue, and intraoperatively detected around the perisentinel node area and dissected were considered SLN. SLN positivity was evaluated by both frozen and final pathology. The patients with at least one SLN (+) were considered SLN (+). The study protocol was approved by the ethics committee of our institution.

\subsection{Statistical Analysis}

The data were analyzed in SPSS software (version 11.5). Descriptive statistics were expressed as mean $\pm S D$ and median (minimum-maximum) for quantitative variables and as the number of patients (percentage) for qualitative variables. Whether there was a difference between more than two categories of a qualitative variable, according to a quantitative variable, it was analyzed with the Student's t-test. Furthermore, if normal distribution assumptions were met, it was evaluated using the Mann-Whitney U test; otherwise, it was not analyzed. The Chi-squared test was used to analyze the correlation between two qualitative variables. A p-value less than 0.05 was considered statistically significant.

\section{Results}

The study included a total of 1,050 patients, who were assigned into two groups of surgery $(n=900)$ and NAC $(n=150)$. The patient flow chart is illustrated in Figure 1.

\subsection{Surgery Group}

The mean age of the patients was $54.80 \pm 11.35$ years, and $82(9.1 \%)$ cases were under 40 years of age. Of the patients, 311 (34.5\%), 22 (2.4\%), and 4 $(0.4 \%)$ individuals had SLNM (macrometastasis), micrometastasis, and ITC, respectively. A total of 215 (23.8\%) patients underwent ALND, and the mean number of dissected SLNs was $2.57 \pm 1.24$. The general characteristics of the patients in the surgery group are shown in Table 1.

When the factors affecting SLNM were analyzed, the univariate analyses showed that under 40 years of age, grades II and III, a tumor size of $>2 \mathrm{~cm}$, LVI, Her2 ( $)$, and triple negative (TN) increased the risk of metastasis, while the multivariate analyses showed that grade III, LVI, Her2 (+), and TN increased the risk of metastasis (Table 2). The analyses also revealed LVI as the most important risk factor for SLN metastasis (OR: 7.23; 95\% 


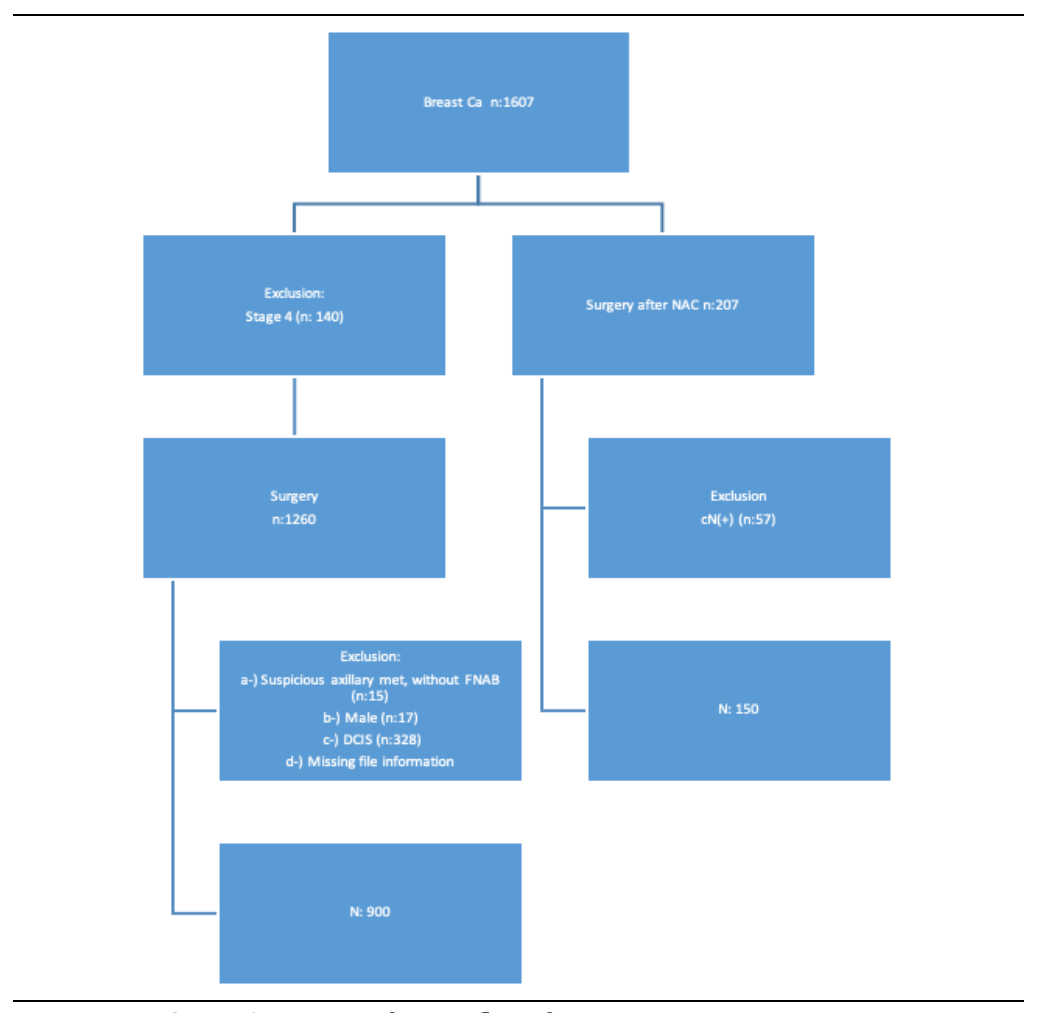

Figure 1. Patient selection flow chart

\begin{tabular}{lcc}
\hline \multicolumn{1}{l}{ Table 1. General characteristics of the patients in the surgery group } & \\
\hline Variables & & \\
\hline & $<40$ & $82(9.1 \%)$ \\
Age, $\mathbf{n}(\%)$ & $40-49$ & $228(25.3 \%)$ \\
& $50-59$ & $275(30.5 \%)$ \\
Menopause, n(\%) & $>=60$ & $315(34.9 \%)$ \\
& Premenopause & $264(29.3 \%)$ \\
& Postmenopause & $634(70.7 \%)$ \\
Tumor location, n(\%) & Central & $31(3.4 \%)$ \\
& Upper outer quadrant & $270(29.9 \%)$ \\
& Lower outer quadrant & $154(17.1 \%)$ \\
& Lower inner quadrant & $117(13 \%)$ \\
T stage, n(\%) & Upper inner quadrant & $198(22 \%)$ \\
& Multicentric & $27(3 \%)$ \\
& T1mic & $8(0.9 \%)$ \\
Histology, n(\%) & T1a & $25(2.5 \%)$ \\
& T1b & $81(8.9 \%)$ \\
Grade, n(\%) & T1c & $290(31.3 \%)$ \\
& T2 & $454(50.2 \%)$ \\
ER, n(\%) & T3 & $42(4.5 \%)$ \\
PR, n(\%) & IDC & $678(75.2 \%)$ \\
Cerb-b2, n(\%) & ILC & $66(7.3 \%)$ \\
LVI, n(\%) & Others & $156(17.3 \%)$ \\
\hline Luminal tip, n(\%) & 1 & $107(11.9 \%)$ \\
& 2 & $406(45 \%)$ \\
& 3 & $381(42.2 \%)$ \\
\hline
\end{tabular}

ER: estrogen receptor, PR: progesterone receptor, LVI: lymphovascular invasion 


\begin{tabular}{|c|c|c|c|c|c|}
\hline & & $\begin{array}{c}\text { Univariate analyses } \\
\text { OR (\%95CI) }\end{array}$ & P-value & $\begin{array}{c}\text { Multivariate analyses } \\
\text { OR (\%95CI) }\end{array}$ & P-value \\
\hline \multirow{2}{*}{ Age } & $<40$ & Reference & & 1 & \\
\hline & $\geq 40$ & $0.71(0.59-0.91)$ & 0.05 & $0.89(0.77-1.33)$ & 0.09 \\
\hline \multirow{3}{*}{ Grade } & I & Reference & & 1 & \\
\hline & II & $2.13(0.82-3.44)$ & 0.02 & $1.61(1.1-2.65)$ & 0.06 \\
\hline & III & $3.46(1.93-4.33)$ & 0.01 & $3.15(2-4.9)$ & 0.01 \\
\hline \multirow{2}{*}{ LVI } & No & Reference & & 1 & \\
\hline & Yes & 7.23 (3.09-9.32) & $<0.001$ & $7.09(3.01-8.87)$ & $<0.001$ \\
\hline \multirow{2}{*}{ Tumor size } & $\leq 2 \mathrm{~cm}$ & Reference & & 1 & \\
\hline & $>2 \mathrm{~cm}$ & $2.17(1.11-3.85)$ & 0.02 & $1.45(1.05-2.17)$ & 0.07 \\
\hline \multirow{4}{*}{ Biological subtype } & Luminal A & Reference & & 1 & \\
\hline & Luminal B & $1.15(0.79-1.28)$ & 0.08 & $1.14(0.83-1.35)$ & 0.08 \\
\hline & Her2(+) & $4.41(2.26-6.6)$ & $<0.001$ & $4.21(2.03-6.23)$ & $<0.001$ \\
\hline & TN & $3.71(2.14-5.5)$ & $<0.001$ & $3.13(2.02-4.98)$ & $<0.001$ \\
\hline
\end{tabular}

LVI: lymphovascular invasion

TN: triple negative

CI: 3.09-9.32). In the surgery group, 109 patients had LVI. The rate of metastasis was $68 \%$ for the patients with LVI, and of the patients with LVI, 62 cases were grade III. The risk of metastasis was $72.5 \%$ for the patients with LVI and grade III. This risk was $75 \%$ for the Her2 (+) patients with LVI, while it was $78.5 \%$ for the TN patients. All Her2 (+) or TN patients with LVI were grade III.

\subsection{Neoadjuvant Therapy Group}

The NAC group included 150 patients, and the mean age of the patients was $43.0 \pm 2.1$ years with a mean body mass index (BMI) of $28 \pm 1.2$. The characteristics of these patients are shown in Table 3. Of 150 patients who received NAC, 40 and 110 cases were $\mathrm{cN} 0$ and $\mathrm{cN}(+)$, respectively, while 12 patients with cN0 after NAC were SLN (+), and 77 cases with $\mathrm{cN}(+)$ were found to be SLN (+).

Of these patients, $73.4 \%$ of them were $\mathrm{cN}(+)$ in the pre-NAC period. SLNM was detected in 89 (59.3\%) patients as a result of the SLNB. The rate of SLNM was $30 \%(12 / 40)$ in the cN0 patients, while it was $70 \%$ $(77 / 110)$ in the $\mathrm{cN}(+)$ patients, and the mean number of dissected SLNs was $2.27 \pm 1.36$. Despite a post-NAC nodal metastasis rate of $59.3 \%$, the rate of the patients who underwent ALND was $74 \%(n=111)$. Although 22 patients were SLNB (-), they underwent ALND. The reasons for this were the thought of an insufficient number of dissected SLN, an intraoperative SLN suggestive of metastasis, and routine ALND performance on patients who had received NAC before clinical standard practices developed. Of the patients in the NAC group, 40 (26\%) cases were found to have a clinical complete response and 110 (74\%) individuals were found to have a partial response.

The univariate analyses revealed an age of $>60$ years, grade I, LVI, clinical findings of LN metastasis in the pre-NAC period $\mathrm{cN}(+)$ and luminal A subtype as factors affecting post-NAC SLNM, while the multivariate analyses showed pre-NAC clinical findings of LN metastasis and luminal A subtypes as effective factors. The risk of SLNM was 30\% (12/40) for the patients with $\mathrm{cN0}$, and it was $70 \%$ for the patients with $\mathrm{cN}(+)(77 / 110)$. The risk of SLNM was $77.4 \%$ for the $\mathrm{cN}(+)$ luminal A patients (Table 4 ).

\begin{tabular}{lcc}
\hline \multicolumn{1}{l}{ Table 3. General characteristics of the patients in the neoadjuvant chemotherapy group } \\
\hline Variables & & \\
\hline Menopause, n (\%) & Premenopause & $106(70 \%)$ \\
& Postmenopause & $44(30 \%)$ \\
T stage, n (\%) & T1 & $4(2.6 \%)$ \\
& T2 & $44(29.3 \%)$ \\
& T3 & $64(42.6 \%)$ \\
Node, n (\%) & T4 & $38(25.3 \%)$ \\
& Positive & $40(26.6 \%)$ \\
Grade, n (\%) & Negative & $110(73.4 \%)$ \\
& 1 & $6(4 \%)$ \\
& 2 & $50(33 \%)$ \\
Luminal type, n (\%) & 3 & $94(63 \%)$ \\
& Luminal A-B & $78(52 \%)$ \\
& Her 2 positive & $38(25 \%)$ \\
\end{tabular}




\begin{tabular}{|c|c|c|c|}
\hline & & OR (\%95 CI) & P-value \\
\hline \multirow{4}{*}{ Age } & $>60$ & Reference & \\
\hline & $<40$ & $0.84(0.64-0.89)$ & 0.06 \\
\hline & $40-49$ & $0.90(0.71-0.92)$ & 0.2 \\
\hline & $50-59$ & $0.94(0.85-1.08)$ & 0.35 \\
\hline \multirow{3}{*}{ Grade } & I & $1.27(1.05-1.33)$ & 0.1 \\
\hline & II & $1.04(0.93-1.12)$ & 0.55 \\
\hline & III & Reference & \\
\hline \multirow{2}{*}{ LVI } & No & Reference & \\
\hline & Yes & $1.13(1.09-1.32)$ & 0.08 \\
\hline \multirow{2}{*}{ Pre-NAC node status } & cNO & Reference & \\
\hline & $\mathrm{cN}(+)$ & $4.67(4.1-5.98)$ & $<0.001$ \\
\hline \multirow{4}{*}{ Biological subtype } & Luminal A & Reference & \\
\hline & Luminal B & $0.81(0.77-1.28)$ & 0.05 \\
\hline & Her2(+) & $0.61(0.36-1.6)$ & $<0.001$ \\
\hline & TN & $0.77(0.39-1.56)$ & $<0.001$ \\
\hline
\end{tabular}

LVI: lymphovascular invasion

TN: triple negative

\section{Discussion}

The status of ALN still remains the most important prognostic and predictive value in the treatment of invasive breast cancer. The status of ALN also determines the extent of axillary surgery $(1,8)$. It is known that patients with SLNB $(-)$ do not benefit from ALND. Moreover, the NSABP B-32 study showed that ALND was unnecessary in patients with micro-metastasis and ITC in SLN (9). With the demonstration that ALND is not necessary for all patients with metastases detected by SLNB, a new era has begun in the surgical treatment of breast cancer $(10,11)$. At the end of these studies, it has been understood that the concepts of SLNM and ALNM are different entities.

The SLNM rates vary between $27 \%$ and $35 \%$ among patients undergoing surgery without NAC. It can be speculated that approximately $2 / 3$ of patients with early-stage breast cancer undergoing SLNB will have negative SLNB $(12,13)$. Considering the ACOSOG Z0011 study criteria, the necessity of ALND disappears in approximately half of the patients with SLNM. The next step is to question the necessity of axillary staging (i.e., SLNB). For this purpose, the results of a multicenter prospective randomized SOUND study comparing pathological and radiological staging of the axilla may be guiding (14). If low-risk groups are identified for SLNM, SLNB may not be a routine intervention since complications, such as lymphedema, nerve injury, and articular movement disorders have been reported for SLNB $(15,16)$.

Age, menstrual cycle, BMI, tumor size, grade, LVI, HR, Ki-67 prognostic index, HER2/neu, tumor location, multifocality, and neoadjuvant treatment characteristics are among the parameters, the relationship of which with SLNM has been investigated. In these studies, LVI draws attention as the most strongly associated feature with SLNM. Malter et al. have determined LVI together with the tumor size as the factor affecting SLNM the most. In this series, the SLNM rate was found to be $75 \%$ in patients with LVI (17). In our series, LVI appeared to be the most effective factor, and our SLNM rate was $68 \%$ in our patients with LVI. Extracapsular invasion is known to be an important factor for non-sentinel metastases $(18,19)$. The spread of tumor cells out of the LN capsule may explain metastasis from the lymph node to another lymph node; however, theoretically, tumor cells must invade into the lymph ducts for spread from the primary tumor mass to SLN. Experimental and clinical studies have shown that newly formed lymph channels around the tumor trigger lymphatic metastasis (20).

In their study, Fujii et al. suggested that LVI increased the risk of SLNM up to eight times (18). LVI may primarily be a characteristic of tumors that tend to spread regionally. Furthermore, LVI has been defined as a parameter that shortens overall survival $(21,22)$. There are numerous studies demonstrating the strong relationship between regional metastasis and LVI (23). In these studies, it is notable that the metastasis potential of LVI increases as the tumor size increases. In our study, it was shown that together with LVI, the biological characteristics (grade and receptor status) of the tumor rather than the size of the tumor determined SLNM. Nevertheless, it can be speculated that the risk of SLNM was very high in the patients with LVI, and patients with LVI should not be included in this group when identifying patients who would not require SLNB.

The rate of Her2 positivity is over $25 \%$ in patients with breast cancer. In their study published in 2020, Diotaiuti et al. evaluated the relationship of biological subtypes with SLNM and found that the Her2 (+) group had the highest risk of SLNM, while the luminal A subtype group had the lowest risk of SLNM. While the rate of SLNM was $40 \%$ in Her2 (+) tumors between 2 and $5 \mathrm{~cm}$, this rate was found to be $10 \%$ in Her2 (-) tumors (24). In our study, Her2 (+) appeared to be the most strongly correlated parameter after 
LVI. Among the biological subtypes, the Her2 (+) group was found to be ranked first with a SLNM rate of $49 \%$. It should be kept in mind that the status of Her2 has been unknown in an important group of patients in studies showing no relationship between Her2 status and SLNM (25).

It has been suggested that as a behavioral pattern, TN tumors are more prone to systemic metastasis rather than regional metastasis (26). Though it has been argued that TN tumors do not have an increased risk for SLNM, regional metastasis rates are relatively high in these tumors due to their aggressive features (26-28). It should also be kept in mind that hormone receptor negativity, just like $\mathrm{TN}$, is effective in determining the pattern of metastasis (29). In our study, TN was found to be a factor that increased SLN metastasis, even though it was not as strong as Her2 $(+)$. In the series of Ding et al., grade was found to be one of the three factors affecting SLNM most. In this study, the rate of SLNM was $67 \%$ in grade III patients younger than 40 years with a tumor larger than $2 \mathrm{~cm}$ (30). Moreover, in our series, this risk was $72.5 \%$ for the patients with LVI and grade III characteristics. The fact that all of our Her2 ( ) or TN patients with LVI were grade III is remarkable in terms of reflecting the aggressiveness determination of the grade III feature.

Younger patient age has been associated with SLNM in many studies (31). They mostly have high Ki-67, HR (-), Her2 (+), and grade III features (32). In our study, under 40 years of age was found to be a risk factor in the univariate analyses, while this feature was not significant in the multivariate analyses, and the biological features remained in the foreground. Increasing tumor size has also appeared to be a risk factor for SLNM in many studies. The risk of SLNM in T1a tumors has been reported around $10 \%$ (33). When the tumor size increases from $10 \mathrm{~mm}$ to $25 \mathrm{~mm}$, this rate increases from $11 \%$ to $36 \%$ (34). In our study, the tumor size, which had a significant effect in the univariate analyses, lost its significance in the multivariate analyses, just like the grade.

It has been argued that upper outer quadrant tumors and multifocal tumors will metastasize more frequently due to their location and proximity to the axilla (11). Biological features rather than physical characteristics come to the fore in metastasis. Tumor localization and multifocality have not been found to be significant parameters in many studies on this subject, as well as in our study. At least low-risk patients for SLNM can be identified by evaluating all these factors. Radiological examinations may be preferred instead of surgical intervention for axillary staging in patients with a morbidity risk in terms of SLNB or anesthesia and in the low-risk group for metastasis. Knowing the low-risk patient group can also guide us in surgical planning. Assuming that lowrisk patients will not receive adjuvant RT, simultaneous autologous reconstructions may be recommended to these patients. High-risk patients can be referred to as late reconstruction in the foreground.

ALND was performed on patients with $\mathrm{cN}(+)$ conventionally in the pre-NAC period and routinely in the post-NAC period (35). With a better understanding of the biological behavior of breast cancer, NAC has been used more frequently for treatment, and the prevention of ALND has become an important goal of NAC. The safety of SLNB has been established in patients who remained postNAC cN0 or regressed to cN0 (36). Today, the necessity of ALND in patients with post-NAC SLN metastasis has been questioned (37). In general, it can be said that tumors with parameters determining their aggressive behavior respond best to NAC. With the knowledge of these factors, a considerable increase has been found in the rate of patients receiving NAC, and a significant number of patients have avoided ALND. As the clinical contribution of ALND is nowadays questioned in patients with SLNM who have received NAC, the factors affecting post-NAC SLN metastasis may guide the treatment planning. In our study, the univariate analyses revealed an age of $>60$ years, grade I, LVI, nodal metastasis in the pre-NAC period, and luminal A subtypes as factors affecting postNAC SLN metastasis. The multivariate analyses, on the other hand, showed nodal metastasis in pre-NAC as the most effective factor. The other effective factor was the luminal A subtype. Although the probability of SLNB (-) (yPN0) has been found to be $78 \%$ in pre-NAC cN0 patients, this rate has been found to be $35 \%$ in $\mathrm{cN}(+)$ patients (38). The effect of patient age in terms of regional response in $\mathrm{cN}(+)$ patients has been found to be more significant than that in cN0 patients. Since there were only 12 patients with SLNM in the cN0 patient group, factors affecting these two groups could not be evaluated in our series. The biological features of the tumor determine the treatment response in patients receiving NAC; nonetheless, it can be speculated that the main determinant of the SLNM risk is the preNAC regional stage. In the light of this information, simultaneous reconstruction can be recommended for patients with $\mathrm{CNO}$ and predicted to be yPNO with a high probability, assuming that they will not receive RT. SLNB may not be performed on comorbid patients in this group. For patients with $\mathrm{cN}(+)$ and a low probability of yPN0, reconstruction is planned in the late period, and false negativity rates can be attempted to be reduced by dissecting more SLNs.

Regarding the limitations of this study, one can refer to its retrospective nature. Moreover, the clinical axillary positivity in patients who received NAC was not pathologically verified by FNAB in all patients. Additionally, primary tumor response in the patients who received NAC was not measured 
objectively in the preoperative period using a standard radiological technique.

\section{Conclusion}

Knowing the factors affecting SLNM can provide clues for the type of intervention, reconstruction, and RT planning of patients to be operated directly or after NAC. In our study, it was found that patient age, tumor size, tumor biology, tumor grade, and especially LVI status were very important in predicting SLN positivity. It is believed that these features should be taken into account when determining the treatment strategy.

\section{Footnotes}

Ethical Approval: The study protocol was approved by the ethics committee of the University of Health Sciences, Ankara Abdurrahman Yurtaslan Oncology Training and Research Hospital, Ankara, Turkey.

Funding/Support: No

Financial Disclosure: No

Conflicts of Interest: The authors had no conflict of interest to declare.

\section{References}

1. Giuliano AE, Kirgan DM, Guenther JM, Morton DL. Lymphatic mapping and sentinel lymphadenectomy for breast cancer. Annals of surgery. 1994;220(3):391-8; discussion 8-401. doi: 10.1097/00000658-199409000-00015

2. Krag DN, Weaver DL, Alex JC, Fairbank JT. Surgical resection and radiolocalization of the sentinel lymph node in breast cancer using a gamma probe. Surgical oncology. 1993;2(6):335-9; discussion 40. .doi: 10.1016/0960-7404(93)90064-6

3. Giuliano AE, Ballman KV, McCall L, Beitsch PD, Brennan MB, Kelemen PR, et al. Effect of axillary dissection vs no axillary dissection on 10-year overall survival among women with invasive breast cancer and sentinel node metastasis: the ACOSOG Z0011 (Alliance) randomized clinical trial. Jama. 2017;318(10):918-26. doi:10.1001/jama.2017.11470

4. Golshan M, Loibl S, Wong SM, Houber JB, O'Shaughnessy J, Rugo HS, et al. Breast Conservation After Neoadjuvant Chemotherapy for Triple-Negative Breast Cancer: Surgical Results From the BrighTNess Randomized Clinical Trial. JAMA surgery. 2020;155(3):e195410. doi: 10.1001/jamasurg.2019.5410

5. Tadros AB, Yang WT, Krishnamurthy S, Rauch GM, Smith BD, Valero V, et al. Identification of Patients With Documented Pathologic Complete Response in the Breast After Neoadjuvant Chemotherapy for Omission of Axillary Surgery. JAMA surgery. 2017;152(7):665-70. doi: 10.1001/jamasurg.2017.0562

6. Spring LM, Fell G, Arfe A, Sharma C, Greenup RA, Reynolds KL, et al. Pathological complete response after neoadjuvant chemotherapy and impact on breast cancer recurrence and survival: a comprehensive meta-analysis. Clinical Cancer Research. 2020;26(12):2838-2848 doi: 10.1158/10780432.CCR-19-3492

7. Mitchell RB, Archer SM, Ishman SL, Rosenfeld RM, Coles S, Finestone SA, et al. Clinical Practice Guideline: Tonsillectomy in Children (Update). Otolaryngol Head Neck Surg. 2019;160(1_suppl):S1-S42.

8. Luini A, Gatti G, Ballardini B, Zurrida S, Galimberti V, Veronesi $\mathrm{P}$, et al. Development of axillary surgery in breast cancer. Annals of oncology : official journal of the European Society for Medical Oncology. 2005;16(2):259-62. doi: 10.1177/0194599818801757

9. Krag DN, Anderson SJ, Julian TB, Brown AM, Harlow SP, Costantino JP, et al. Sentinel-lymph-node resection compared with conventional axillary-lymph-node dissection in clinically node-negative patients with breast cancer: overall survival findings from the NSABP B-32 randomised phase 3 trial. The lancet oncology. 2010;11(10):927-33. doi: 10.1016/S14702045(10)70207-2

10. Giuliano AE, Hunt KK, Ballman KV, Beitsch PD, Whitworth PW, Blumencranz PW, et al. Axillary dissection vs no axillary dissection in women with invasive breast cancer and sentinel node metastasis: a randomized clinical trial. Jama. 2011;305(6):569-75. doi: 10.1001/jama.2011.90

11. Tvedskov TF, Jensen M-B, Ejlertsen B, Christiansen P, Balslev E, Kroman $\mathrm{N}$ et al. Prognostic significance of axillary dissection in breast cancer patients with micrometastases or isolated tumor cells in sentinel nodes: a nationwide study. Breast cancer research and treatment. 2015;153(3):599-606. doi: https://doi.org/10.1007/s10549-015-3560-7

12. Viale G, Zurrida S, Maiorano E, Mazzarol G, Pruneri G, Paganelli G, et al. Predicting the status of axillary sentinel lymph nodes in 4351 patients with invasive breast carcinoma treated in a single institution. Cancer. 2005;103(3):492-500. doi: 10.1002/cncr.20809

13. Yoshihara E, Smeets A, Laenen A, Reynders A, Soens J, Van Ongeval C, et al. Predictors of axillary lymph node metastases in early breast cancer and their applicability in clinical practice. Breast (Edinburgh, Scotland). 2013;22(3):357-61. doi:10.1016/i.breast.2012.09.003

14. Gentilini O, Veronesi U. Abandoning sentinel lymph node biopsy in early breast cancer? A new trial in progress at the European Institute of Oncology of Milan (SOUND: Sentinel node vs Observation after axillary UltraSouND). The Breast. 2012;21(5):678-81. doi: 10.1016/j.breast.2012.06.013

15. Sackey H, Johansson H, Sandelin K, Liljegren G, MacLean G, Frisell J, et al. Self-perceived, but not objective lymphoedema is associated with decreased long-term health-related quality of life after breast cancer surgery. European journal of surgical oncology : the journal of the European Society of Surgical Oncology and the British Association of Surgical Oncology. 2015;41(4):577-84. doi: 10.1016/i.ejso.2014.12.006

16. Sackey H, Magnuson A, Sandelin K, Liljegren G, Bergkvist L, Fülep Z, et al. Arm lymphoedema after axillary surgery in women with invasive breast cancer. The British journal of surgery. 2014;101(4):390-7. doi:10.1002/bjs.9401

17. Malter W, Hellmich M, Badian M, Kirn V, Mallmann P, Krämer $S$, et al. Factors Predictive of Sentinel Lymph Node Involvement in Primary Breast Cancer. Anticancer research. 2018;38(6):3657-62. doi: 10.21873/anticanres. 12642

18. Fujii T, Yajima R, Yamaguchi S, Yanagita Y, Fujisawa T, Hirakata $\mathrm{T}$, et al. Extracapsular invasion of sentinel lymph nodes is not associated with disease recurrence in breast cancer. International surgery. 2014;99(4):305-8. doi: 10.9738/INTSURG-D-13-00012.1

19. Yajima R, Fujii T, Yanagita Y, Fujisawa T, Miyamoto T, Hirakata $\mathrm{T}$, et al. Prognostic value of extracapsular invasion of axillary lymph nodes combined with peritumoral vascular invasion in patients with breast cancer. Annals of surgical oncology. 2015;22(1):52-8. doi: 10.1245/s10434-014-3941-x

20. Achen MG, Stacker SA. Molecular control of lymphatic metastasis. Annals of the New York Academy of Sciences. 2008;1131(1):225-34. doi:10.1196/annals.1413.020

21. Ejlertsen B, Jensen M-B, Rank F, Rasmussen BB, Christiansen $P$, Kroman N, et al. Population-based study of peritumoral lymphovascular invasion and outcome among patients with operable breast cancer. JNCI: Journal of the National Cancer Institute. 2009;101(10):729-35. doi: 10.1093/inci/djp090

22. Rakha EA, Martin S, Lee AH, Morgan D, Pharoah PD, Hodi Z, et al. The prognostic significance of lymphovascular invasion in invasive breast carcinoma. Cancer. 2012;118(15):3670-80. doi: $10.1002 /$ cncr.26711

23. Cornwell LB, McMasters KM, Chagpar AB. The impact of 
lymphovascular invasion on lymph node status in patients with breast cancer. The American surgeon. 2011;77(7):874-7. doi:10.1177/000313481107700722

24. Diotaiuti S, De Summa S, Altieri R, Dantona C, Tommasi S, Di Gennaro $\mathrm{M}$, et al. Biomarker phenotyping drives clinical management in axillary sentinel node: A retrospective study on women with primary breast cancer in 2002. Oncology letters. 2020;20(3):2469-76. doi: 10.3892/ol.2020.11793

25. Ozemir I, Orhun K, Eren T, Baysal H, Sagiroglu J, Leblebici M, et al. Factors affecting sentinel lymph node metastasis in Turkish breast cancer patients: Predictive value of Ki- 67 and the size of lymph node. Bratislavske lekarske listy. 2016;117(8):436-41. doi: $10.4149 /$ bll 2016085.

26. Ugras S, Stempel M, Patil S, Morrow M. Estrogen receptor, progesterone receptor, and HER2 status predict lymphovascular invasion and lymph node involvement. Annals of surgical oncology. 2014;21(12):3780-6. doi: 10.1245/s10434-014-3851-y

27. Crabb SJ, Cheang MC, Leung S, Immonen T, Nielsen TO, Huntsman DD, et al. Basal breast cancer molecular subtype predicts for lower incidence of axillary lymph node metastases in primary breast cancer. Clinical breast cancer. 2008;8(3):249-56. . doi: 10.3816/CBC.2008.n.028

28. Gangi A, Mirocha J, Leong T, Giuliano AE. Triple-negative breast cancer is not associated with increased likelihood of nodal metastases. Ann Surg Oncol. 2014;21(13):4098-103. doi: 10.1245/s10434-014-3989-7

29. Mattes MD, Bhatia JK, Metzger D, Ashamalla H, Katsoulakis E. Breast Cancer Subtype as a Predictor of Lymph Node Metastasis according to the SEER Registry. Journal of breast cancer. 2015;18(2):143-8. doi: 10.4048/ibc.2015.18.2.143

30. Ding J, Jiang L, Wu W. Predictive Value of Clinicopathological Characteristics for Sentinel Lymph Node Metastasis in Early Breast Cancer. Medical science monitor : international medical journal of experimental and clinical research. 2017;23:4102-8. . doi:10.12659/MSM.902795

31. Mustafa IA, Cole B, Wanebo HJ, Bland KI, Chang HR. The impact of histopathology on nodal metastases in minimal breast cancer. Archives of surgery (Chicago, Ill : 1960). 1997;132(4):384-90 doi: 10.1001/archsurg.1997.01430280058008
32. Rivadeneira DE, Simmons RM, Christos PJ, Hanna K, Daly JM, Osborne MP,et al. Predictive factors associated with axillary lymph node metastases in T1a and T1b breast carcinomas: analysis in more than 900 patients. Journal of the American College of Surgeons. 2000;191(1):1-6; discussion -8. doi: 10.1016/s1072-7515(00)00310-0

33. Silverstein MJ, Skinner KA, Lomis TJ. Predicting axillary nodal positivity in 2282 patients with breast carcinoma. World journal of surgery. 2001;25(6):767-72. doi: : 10.1007/s00268001-0003-x

34. Cutuli B, Velten M, Martin C. Assessment of axillary lymph node involvement in small breast cancer: analysis of 893 cases. Clinical breast cancer. 2001;2(1):59-65. doi: 10.3816/CBC.2001.n. 012

35. Mittendorf EA, Caudle AS, Yang W, Krishnamurthy S, Shaitelman S, Chavez-MacGregor M, et al. Implementation of the american college of surgeons oncology group z1071 trial data in clinical practice: is there a way forward for sentinel lymph node dissection in clinically node-positive breast cancer patients treated with neoadjuvant chemotherapy?. Annals of surgical oncology. 2014;21(8):2468-73. doi: 10.1245/s10434014-3775-6

36. Boileau J-F, Poirier B, Basik M, Holloway CM, Gaboury L, Sideris L, et al. Sentinel node biopsy after neoadjuvant chemotherapy in biopsy-proven node-positive breast cancer: the SN FNAC study. Journal of Clinical Oncology. 2015;33(3):258-64. doi: 10.1200/JC0.2014.55.7827

37. de Boniface J, Frisell J, Andersson Y, Bergkvist L, Ahlgren J, Rydén L, et al. Survival and axillary recurrence following sentinel node-positive breast cancer without completion axillary lymph node dissection: the randomized controlled SENOMAC trial. Bmc Cancer. 2017;17(1):1-7. doi:10.1186/s12885-017-3361-y

38. Murphy BL, Hoskin TL, Day C, Habermann EB, Boughey JC. Preoperative prediction of node-negative disease after neoadjuvant chemotherapy in patients presenting with nodenegative or node-positive breast cancer. Annals of Surgical Oncology. 2017;24(9):2518-25. doi: 10.1245/s10434-017. $\underline{5872-9}$ 